\title{
Discussion on the Differences between Theory and Practice in Vocal Music Teaching
}

\author{
Lei Fu
}

Yangtze University School of Arts, Jingzhou, Hubei, China

\begin{abstract}
We are living in the best era. At present, the economy is growing steadily, and science and technology have driven the rapid development of all walks of life. Correspondingly, art education has received more and more attention from the society, and the teaching of vocal music has also received the attention and love of parents and students. With the continuous replacement of the development concept of the times, the methods of vocal music teaching are also constantly improving. From the transition from the teacher as the main body to the student as the foundation, the teaching emphasizes the development of students and pays more attention to changes in academic conditions, and pays more attention to the combination of theory and practice. And it will find out the difference between reality and theory based on the actual situation, make targeted analysis, find out the shortcomings, formulate a more scientific teaching method, improve teaching mode, improve teaching quality, so as to better improve students' vocal level. This article analyzes the differences between theory and practice in vocal music teaching, and proposes corresponding solutions for reference.
\end{abstract}

Key words: vocal teaching; theory and practice; difference exploration

\section{Introduction}

With the vigorous development of the art industry and the diversification of art forms, vocal music education is therefore very popular. Driven by the progress and development of the times, the content and scope of vocal music teaching have also been continuously expanded, and more outstanding artistic talents have also been cultivated. But overall, there are still some differences in current vocal music teaching, especially between theory and practical experience. For example, there is the problem of a certain degree of separation between singing practice and teaching theory, and the problem of insufficient vocal music culture excavation. This restricts the improvement of vocal music teaching quality to a certain extent. Based on this, we need to conduct an in-depth analysis of the causes of theories and differences in teaching, find out the shortcomings, and improve the teaching methods and teaching modes according to the shortcomings, so as to promote the integration of theory and practice and continuously improve the quality of vocal music teaching.

\section{Analysis of the Differences between Theory and Practice in Vocal Music Teaching}

2.1 Vocal music theory pays more attention to rational expression

Theory and practice are two important components in vocal music teaching. Theory without practice is not enough, and practice without theoretical support is even more incomplete. Therefore, theory and practice are complementary and inseparable. Theory guides practice and practice reflects theory. Only in this way can high-quality vocal expression be presented. Since theory and practice exist as two parts, the difference is inevitable. In the teaching of vocal music, the

Copyright (C) 2021 by author(s) and Frontier Scientific Research Publishing Inc.

This work is licensed under the Creative Commons Attribution International License (CC BY 4.0).

http://creativecommons.org/licenses/by/4.0/ 
theoretical point of view highlights the rational expression of vocal music, focusing on the explanation and indoctrination of vocal principles, which are no temperature and immutable. The focus of practical teaching is emotional expression and the use of skills, such as the use and control of vocalization, intonation, and timbre. At the same time, vocal music practice teaching also emphasizes the penetration and expression of emotions, which is why different vocal music works and different people can perform different effects, and is also the fundamental reason why they give people different feelings. Practitioners themselves have different understandings of vocal music works, and their betting emotions are also different, and the atmosphere and charm presented by works with different emotions naturally have certain differences. Good vocal music works first of all impress the singer himself, and it has a warmth. Only by putting one's own emotions into the work, can they better impress others, so as to reach the highest state of art, and fully embody the value of art.

2.2 Vocal music theory more prominent spiritual perception

Although the theoretical teaching of vocal music is more aimed at the transmission of music theory viewpoints and the preparation of emotions, which is a manifestation of physiological behavior, in the actual interpretation process, the information absorbed by different singers is different, and the spiritual understanding formed is also different. The process of practice is dynamic. In addition to the emotional pavement of theory, practice is a process of expressing, and is the full play of theory. Although on the surface the difference between theory and practice is difficult to understand, it is not true. Practice is to control the voice and vocal cords through the shape of the mouth, and to integrate their own perceptions and emotions of the work and express them through appropriate singing techniques, and let the audience feel, understand, and experience it. It completes the transmission process of vocal music theory, and at the same time, it is also a concrete manifestation of students' professional ability.

\subsection{Vocal music theory focuses on stability}

The theoretical knowledge of vocal music is not only for a certain individual, it is equivalent to a standard, and is the basic criterion for every learner of vocal music. The focus of vocal music theory is to highlight the beauty in vocal music, and let the singer pass this beauty through corresponding skills. Therefore, the accumulation of theory is equivalent to a long-term practice process. It controls its own emotions in stability, integrates corresponding theoretical knowledge, and cultivates artistic talents. While practice is a kind of expression with a certain degree of randomness, and it has a certain relationship with the mood, state of mind and environment of the singer at the time. Vocal music practice is more like a process of self-enjoyment by learners. Learners interpret the artistic connotation of the work in practice and integrate their own understanding of the work. At the same time, the emotions of the singer are penetrated, making the artistic conception expressed in the work richer, let the audience to experience a richer vocal beauty.

\section{Analysis of the Relevance of Vocal Music Teaching Theory and Practice}

Theory and practice are inseparable. First of all, the theoretical knowledge of vocal music is summarized from practice, including specific music theory, singing methods, etc. If you trace back from the source, it is impossible to summarize these music theory knowledge without practice. So, in other words, practice is the origin of the theory. Vocal music theory is continuously summarized and integrated according to specific vocal performance forms and performance techniques, and it carries people's good wishes and pursuits for vocal art. At the same time, the formation of vocal music theory also means that this form has been recognized by the majority of the masses, so it has been summarized, sorted and passed down. In this process, human beings are not only learners of vocal music, but also transmitters of vocal music. From another perspective, theory to practice is also a process of harvesting and dissemination. The prerequisite of harvest is that only because of love, love will take the initiative to learn, and will have the willingness to spread after learning results. Therefore, strong interest is the basic premise of learning vocal music. Only by stimulating students' interest can 
we systematically study vocal music. Secondly, vocal music theory and singing practice are based on each other. From the perspective of learning, learning vocal music is to improve personal artistic quality, lay a good professional foundation, and pave the way for subsequent development. This requires a solid theoretical foundation and solid basic skills. Only in this way can the expressive and appealing power of vocal art be fully demonstrated.

\section{An Effective Way to Combine Theory and Practice in Vocal Music Teaching}

4.1 Innovate educational forms to promote the connection between theory and practice

In the process of vocal music teaching, it is far from enough to focus solely on the teaching of theory or the emphasis on practice. The only way to address the current problem of insufficient connection between the two is to improve the teaching methods and continuously innovate teaching methods. To better integrate theory and practice, we should not only pay attention to the transfer of music theory, but also not neglect the cultivation of actual singing ability. For example, creating a good practice display platform or using information technology to promote the integration and cohesion between the two, so that students can understand their own shortcomings in vocal learning through repeated practice, and learn from the relationship between music theory and skills. Starting from connectivity, continuously strengthen and consolidate professional knowledge to obtain better learning results. Based on case analysis, actual vocal music cases can be introduced in vocal music theory education, combined with specific examples to make analysis, and guide students to practice based on cases, thereby combining theoretical learning and practical exercises.

4.2 Relying on the internet and information technology to dig deep into the local vocal characteristics

As mentioned above, the unique art form of vocal music is the long-term wisdom of the vast working people in our country. The expression of vocal music and its artistic connotation in each region are different. In addition to working hard in theory and practice, we must also actively explore local characteristics of vocal music. This will help students' vocal music knowledge to be more diversified, and by absorbing different essence of vocal music, it is more conducive to the integration of vocal theory and technique. For example, teaching the ethnic characteristics of vocal music to students with the background, cultural connotation, emotional expression and other related knowledge of the formation of ethnic vocal music can broaden students' horizons and knowledge, and help them understand the broad and profound culture of the motherland. At the same time, different vocal music cultures can better promote the development of students' artistic literacy.

\subsection{Actively build a professional skills competition platform}

Theoretical education is a process, and true knowledge can only be derived from practice. The construction of the professional skills competition platform is an important prerequisite for realizing the integration of theory and practice. It is more targeted and practical, and more in line with the actual needs of vocal music education. Moreover, the professional skills competition has a certain guiding role in the design of vocal music teaching goals and curriculum system. Based on this, colleges and universities can also be oriented by vocal music competitions, strengthen curriculum planning and research, continuously reform the curriculum system and teaching content, and develop targeted and practical training materials to further highlight the students' vocal theory learning effects and practical ability. At the same time, through diversified teaching evaluation to promote and mobilize the enthusiasm of students to participate in the vocational skills competition, in order to promote the continuous improvement of students' regular course learning and practical operation ability. Specifically, relying on the college, a small skill competition of the colleges and departments can be constructed. You can also build a school-level skills competition based on the campus singing contest. It can also cooperate with other universities to build an inter-school music skills competition. Not only that, but it can also organize students to participate in other professional skill competitions to guide students in effective practice. 


\section{Conclusions}

Art is all-encompassing and has extremely rich forms of expression. The existence of art is to better improve the quality of human life and happiness. Everyone can find spiritual sustenance and emotional resonance in different art forms. Vocal music is an important channel for people to express their emotions. Good vocal music can bring people better feelings. This is also the root of the increasing attention to vocal music teaching. As vocal music educators, in addition to the teaching of theoretical knowledge, we must pay more attention to the consolidation of practice in specific vocal music teaching. By combining the actual forms of current vocal music expressions, we should actively research and pursue the compatibility between theoretical knowledge and singing practice, continuously reform and innovate teaching models and methods, formulate more scientific and targeted vocal music teaching programs, so as to further improve the quality of vocal music education, enhance students' music literacy, and better promote students' all-round development.

\section{Conflicts of Interest}

The author declares no conflicts of interest regarding the publication of this paper.

\section{References}

[1] Shi J. (2020). Analysis and Discussion on the Difference between Theory and Practice in Vocal Music Teaching. Journal of Hubei Open Vocational College, (15): 147-148.

[2] Yan X.X. (2019). Discussion on Vocal Music Teaching Combining Theory and Practice---Comment on The Theory of Vocal Music Singing Art and Appreciation of Classic Works. Journal of the Chinese Society of Education, (5): 133.

[3] Feng L.L. (2019). The Theory and Practice Analysis of Vocal Music Teaching in Colleges. Art Science and Technology, (7): 247.

[4] Sun X.M. (2018). A Probe into the Combination of Theory and Practice in National Vocal Music Teaching. Youth Years, (11): 122.

[5] $\mathrm{Wu}$ Y. (2019). Explore the Importance of Emotion in the Teaching of Vocal Music Singing. Heilongjiang Education (Theory \& Practice), (3): 25-26.

[6] Zhang X. (2019). The Theory and Practice of the Teaching Reform of Popular Singing Major---Taking Shijiazhuang City Art School as an Example. Popular Science, (12): 107. 